\title{
Normas Internacionales de Información Financiera como instrumentos de gestión empresarial y control gerencial: más allá de una función en contabilidad
}

\author{
Nydia Marcela Reyes Maldonado* \\ Fernando Chaparro García* \\ Andrés Guerrero Barajas ${ }^{\star \star *}$
}

Fecha de recibido: 1 de septiembre de 2019

Fecha de aprobado: 3 de diciembre de 2019

Para citar: Reyes Maldonado, N. M., Chaparro García, F., \& Guerrero Barajas, A. (2020). Normas Internacionales de Información Financiera como instrumentos de gestión empresarial y control gerencial: más allá de una función en contabilidad. Revista Universidad \& Empresa, 22(39), 1-25. https://doi.org/10.12804/revistas.urosario.edu.co/

* Contadora pública de la Universidad Autónoma de Bucaramanga (UNAB). Doctora en contabilidad y finanzas de las organizaciones de la Universidad de Zaragoza, España. Docente del Programa Contaduría Pública, UnAB. Directora del grupo de investigación en contabilidad y finanzas: Invesconfin. Correo electrónico: nreyes@unab.edu.co

** Contador público de la Universidad Autónoma de Bucaramanga (UNAB). Doctor en ciencias económicas y empresariales de la Universidad de Zaragoza, España. Director del Programa Contaduría Pública, unAB. Integrante del grupo de investigación Invesconfin. Correo electrónico: fchaparr@unab.edu.co

*** Contador público de la Universidad Santo Tomás. Especialista en finanzas de la unAB. Magíster en administración de empresas de la UNAB. Jefe de Crédito y Cartera de la Cooperativa Multiactiva de la Universidad Autónoma de Bucaramanga. Correo electrónico: aguerrero564@unab.edu.co 


\title{
Resumen
}

Los gerentes de las empresas juegan un rol fundamental en la aplicación exitosa de las Normas Internacionales de Información Financiera (NIIF). Utilizar las NIIF como instrumento de gestión y control debería ser algo evidente, pero al parecer su uso se sigue limitando a fines contables. En efecto, esta situación ha llevado a estudiar la percepción que tienen los gerentes colombianos respecto a sus responsabilidades frente a las NIIF y si aceptan que estas se usen para evaluar su gestión. En este sentido, se procedió a diseñar un instrumento que considerara todas las responsabilidades relacionadas con la administración de las entidades contenidas en las NIIF. De este análisis surgieron 33 variables que fueron puestas a consideración de 100 gerentes, quienes mediante una escala de Likert dieron su opinión. Utilizando un análisis factorial se determinaron 5 grupos de variables que obedecen a las principales responsabilidades de la gerencia frente a las NIIF, en los que los resultados mostraron que los gerentes entrevistados no ejercen la gran mayoría de sus funciones bajo las normas y que están en desacuerdo con que estas se utilicen para evaluar la gestión realizada de los recursos de la entidad.

Palabras Clave: NIIF; control; gestión; transparencia.

\section{International Financial Reporting Standards as Instruments of Entrepreneurial and Control Management: Beyond an Accounting Function}

\begin{abstract}
The managers of companies play a fundamental role in the successful application of the International Financial Reporting Standards (IFRS). Using the IFRS as an instrument of control and management should be evident, but it seems their use is still limited to accounting purposes. In fact, this situation has led to the further study of the perception that Colombian managers have about their liability, and if they really accept that, the IFRS will help to evaluate the managers' performance. Based on this, we proceeded to design a tool that considerate all the responsibilities that are related to the administration of companies, included in the IFRs. From this analysis, 33 variables were tested by a hundred managers, who gave their feedback, according to a Likert Scale. Using a factor analysis, five groups of variables were set, they correspond to the main managerial responsibilities towards the IFRS. The results displayed that the managers interviewed do not exert most of their functions under the IFRS and they disagree with using them to evaluate the management of the entity's resources.
\end{abstract}

Keywords: IFRS; control; management; transparency.

\section{Normas Internacionais de Informação Financeira como instrumentos de gestão empresarial e controle gerencial: para além de uma função em contabilidade}

\section{Resumo}

Os gerentes das empresas têm um papel fundamental na aplicação bem-sucedida das Normas Internacionais de Informação Financeira. Utilizar as NIIF como instrumento de gestão e controle deveria ser algo evidente, mas aparentemente seu uso segue-se limitando a fins contáveis. Efetivamente, esta situação tem levado a estudar a percepção que têm os gerentes colombianos respeito a suas responsabilidades frente às NIIF e se aceitam que elas se usarão para avaliar sua gestão. Neste sentido, precedeu-se a desenhar um instrumento que considerara todas as responsabilidades que estão relacionadas com a administração das entidades contidas nas NIIF. Desta análise surgiram 33 variáveis que foram levadas a ser consideradas por 100 gerentes quem através de uma escala de Likert deram sua opinião. Utilizando uma análise fatorial se determinaram 5 grupos de variáveis que obedecem às principais responsabilidades da gerência frente às NIIF, onde os resultados mostraram que os gerentes entrevis- 
tados não exercem a maioria de suas funções sob as normas e que estão em desacordo com que as mesmas se utilizem para avaliar a gestão realizada dos recursos da entidade.

Palavras-chave: NIIF; Controle; Gestão; Transparência.

\section{Introducción}

La convergencia a Normas Internacionales de Información Financiera (NIIF) busca que la información base para la toma de decisiones económicas sea comparable, de tal forma que inversores, prestamistas y otros acreedores puedan tener más y mejores elementos para otorgar recursos a las entidades, para que estas puedan crecer, consolidarse y generar valor. Colombia no fue ajena a este proceso, el cual se llevó a cabo gracias a la Ley 1314 de 2009, tanto en empresas multinacionales como en pequeñas y medianas. Sin embargo, al parecer, los entes no entendieron que la convergencia obedece a todo un cambio en el paradigma empresarial, pues el éxito de su implementación radica en que las empresas desarrollen una cultura de gestión basada en información real, así como en la transparencia y rendición de cuentas sobre cómo ha sido el manejo que los administradores han dado a los recursos que les fueron entregados para su gestión.

En este sentido, el Consejo de Normas Internacionales de Contabilidad (IASB por sus siglas en inglés) en el año 2015 publicó un proyecto de modificación del marco conceptual para la información financiera, cuyo propósito fue poner de manifiesto la necesidad de incluir la administración como un factor importante en el desarrollo de la convergencia a NIIF. En principio se tenía a la contabilidad simplemente como un instrumento de toma de decisiones para los inversores dentro de una organización, pero este punto de vista no fue suficiente, porque también fue fundamental aplicar parámetros de evaluación de la gerencia en la dinámica de su gestión. Por tal motivo, el marco conceptual para la información financiera en su propuesta de actualización llevó más allá el objeto de las NIIF al convertirlas no solo en un medio decisorio al invertir, sino en un instrumento para controlar la gestión de los recursos por parte de la administración de una entidad (Consejo de Normas Internacionales de Contabilidad, 2015).

Jiménez (2010) señala que la globalización e integración económica mundial han obligado a las organizaciones a buscar métodos, procesos y tecnologías innovadoras 
que permitan obtener ventajas comparativas y competitivas en el contexto de la libre competencia, por lo que las organizaciones deben tomar las NIIF como una herramienta gerencial y a su vez como factor de toma de decisiones para dejar de lado el mito de que este tema es concerniente únicamente al área contable y al profesional que lo encabeza.

Un proceso de convergencia a NIIF exitoso requiere como insumo fundamental que la gerencia esté involucrada en todo el proceso, en especial, en el de adaptarlas al nuevo ambiente contable y financiero (Ocampo \& Astudillo, 2015). Así mismo, las NIIF mencionan una cantidad importante de roles de la gerencia frente a la aplicación de las normas, tales como su responsabilidad en la definición de políticas contables, la elaboración de juicios de materialidad, la promoción de la transparencia y la rendición de cuentas, entre otros, lo cual en la práctica podría no estarse ejecutando o delegándose inadecuadamente.

Es por lo anterior que el objetivo de esta investigación parte de establecer la percepción de los directivos especialmente de pequeñas y medianas empresas colombianas sobre el uso de las NIIF como instrumento de gestión y control gerencial teniendo como base los principios administrativos del IASB. En otras palabras, se identificaron todas las responsabilidades que los gerentes tienen en relación con la aplicación de las NIIF y se diseñó un instrumento con ellas, el cual fue puesto a consideración de gerentes con el propósito de establecer si son conocidas por ellos, en qué medida las aplican y si existen dentro de las NIIF funciones claramente definidas de gestión y control. Para lograr lo anterior, se plantearon las siguientes hipótesis de investigación:

Hipótesis nula (1): Los directivos, accionistas y personal encargado del área gerencial, contable y financiera de empresas no hacen aplicables las NIIF en sus procesos de gestión empresarial.

Hipótesis alternativa (1): Los directivos, accionistas y personal encargado del área gerencial, contable y financiera de empresas conocen las NIIF y las aplican en la gestión de sus organizaciones.

Hipótesis nula (2): Los gerentes no están de acuerdo con el objetivo propuesto por el IASB de que las NIIF sirvan para evaluar la gestión de los recursos hecha por la administración. 
Hipótesis alternativa (2): Los gerentes están de acuerdo con el objetivo propuesto por el IASB de que las NIIF sirvan para evaluar la gestión de los recursos hecha por la administración.

\section{Revisión de literatura}

Queda claro que las NIF no pueden limitarse al ámbito contable y financiero, sino que además deben ser contempladas como un sistema de gestión y control en las organizaciones, para lograr de esta forma que la gerencia o el gobierno corporativo sea el que más se beneficie, a través de la generación de nuevos lineamientos y procedimientos que permitan gestionar, informar y controlar, de manera adecuada, los procesos que se realizan tendientes a salvaguardar los activos, mejorar los indicadores financieros y robustecer el sistema de control interno (IFRS Fundation, 2009). Al respecto, para autores como Pineda (2013), al igual que en el ámbito mundial, todas las empresas colombianas requieren estrategias para mantener su competitividad en los mercados nacionales e internacionales y para ello deben adaptarse a las NIIF. Esto implica que desde la administración se le dé importancia a los cambios estructurales de toda la empresa, porque las mismas consideran aún que las NIIF solo modifican el área contable.

Por otra parte, Quintero y Pinzón (2016) precisan que las NIIF le han dado la posibilidad a la gerencia de asumir nuevas responsabilidades que, a su vez, pueden convertirse en oportunidades para su propio beneficio, lo anterior en relación con el concepto de earnings management, entendido como "cualquier práctica llevada a cabo intencionalmente por la gerencia, con fines oportunistas o informativos para reportar los resultados deseados cuando estos discrepan de los reales" (Quintero \& Pinzón, 2016, p. 10). En efecto, uno de los principales argumentos que esbozan estos autores es que la gerencia es juez y parte en la preparación de los estados financieros y tiene la posibilidad de influenciar las políticas, dada su flexibilidad para ajustarse a los resultados que se quieren mostrar de la empresa. Esto genera, a su vez, la oportunidad para que el gerente presente una posición más favorable de la que realmente tiene la empresa. 
Aquí es importante mencionar la escasa relevancia que se le da a dos de los propósitos de la información financiera: mantener a la gerencia controlada y la implementación de la administración del marco conceptual para la información financiera, el cual ayudará a desarrollar normas que permitirán contrarrestar cualquier sesgo que exista en la gerencia, al aplicar las políticas contables de la entidad que informa (Consejo de Normas Internacionales de Contabilidad, 2015).

De hecho, existe una situación que afecta este proceso en cuanto a la toma de decisiones y la relevancia que tiene la información de corte financiero que se obtiene, se trata del conflicto de gerencia. Este surge a partir de la separación entre la propiedad de la empresa y la gestión de la misma y puntualiza la situación en la cual puede haber intereses y objetivos en conflicto, partiendo de la idea de que los directivos y los accionistas poseen intereses opuestos, lo que no permite una correcta toma de decisiones (Lozano, Hidalgo \& Pindado, 2004; Barrios, 2015). Aquí, las NIIF pueden jugar un papel fundamental al tratar de fomentar la mayor transparencia posible en la información suministrada a los usuarios, dando con esto lugar a una reducción del conflicto de agencia, o por lo menos pueden servir como medio disuasorio.

Frente al tema de la implementación de las NIIF como parte de una convergencia contable es imprescindible la responsabilidad de la gerencia no solo como la directa encargada de su implementación, sino por el papel que tiene en la transformación de su gestión, partiendo de las nuevas propuestas financieras y contables que puede asumir. Así las cosas, bajo el proceso de convergencia contable que se suscitó en el país, la actuación del administrador ante los temas contables debe trascender de un ejercicio de observación y delegación de la responsabilidad sobre la información contable hacia una intervención activa, en la que su liderazgo transformacional fomente una nueva cultura en la generación y administración de la información contable en la organización que dirige (Santos, 2015, p. 39).

Es por lo anterior que surgen una serie de interrogantes ante estas situaciones: ¿son conscientes los gerentes de sus responsabilidades bajo los nuevos marcos normativos? ¿Están aplicando los gerentes las NIIF en su gestión? ¿Qué opinión tienen de esta propuesta que involucra utilizar las NIIF para el control y evaluación de su gestión? 


\section{Metodología}

Para esta investigación se utilizó un muestreo no probabilístico por conveniencia que hace referencia a "los casos disponibles a los cuales tenemos acceso" (Hernández, Fernández \& Baptista, 2010, p. 401). Al ser una investigación de tipo exploratorio-descriptivo para un caso específico de los directivos, accionistas y personal encargado del área gerencial, contable y financiera de empresas no resultan convenientes resultados generales, ya que lo que se busca es cómo son acogidas, bajo nuevas circunstancias de mercado, las NIIF. Además, mediante la aplicación de un análisis factorial, se obtuvo una mejor perspectiva de las responsabilidades de la gerencia mediante el análisis de variables latentes no observables directamente.

\subsection{Definición del método de recolección de datos}

La definición del método de recolección de datos tuvo en cuenta la necesidad de diseñar un instrumento confiable y válido. Para ello se eligió un cuestionario que cumpliera con las características necesarias para recopilar la información (Hunt, 1996).

\subsection{Diseño del instrumento}

En la elaboración del instrumento se tuvieron en cuenta los siguientes documentos que se relacionaban directamente con la gestión en la implementación de las NIIF en las empresas: Marco Conceptual para la Información Financiera 2010; NIIF para Pequeñas y Medianas Entidades (NIIF para las PYMES); Comentarios de la gerencia (IFRs Foundation, 2010), y la propuesta del Proyecto Norma Marco Conceptual para la Información Financiera (IFRS Foundation, 2015). El primer paso fue definir las categorías del instrumento con base en los documentos mencionados anteriormente (tablas 1-5).

Tabla 1. Categorías del marco conceptual 2010

\begin{tabular}{|c|c|c|}
\hline \multicolumn{3}{|c|}{ Marco conceptual 2010 (IFRS, 2010) } \\
\hline Categoría & Pregunta(s) en la encuesta relacionada & $\begin{array}{l}\text { Página de } \\
\text { referencia }\end{array}$ \\
\hline $\begin{array}{l}\text { Objetivo } \\
\text { información }\end{array}$ & $\begin{array}{l}\text { ¿La gerencia proporciona información financiera sobre la entidad que es útil a los } \\
\text { inversores, prestamistas y otros acreedores existentes y potenciales para tomar } \\
\text { decisiones sobre el suministro de recursos a la entidad? }\end{array}$ & $\begin{array}{l}\text { Párrafo OB02, } \\
\text { p. } 6\end{array}$ \\
\hline
\end{tabular}




\begin{tabular}{|c|c|c|}
\hline \multicolumn{3}{|c|}{ Marco conceptual 2010 (IFRS, 2010) } \\
\hline Categoría & Pregunta(s) en la encuesta relacionada & $\begin{array}{l}\text { Página de } \\
\text { referencia }\end{array}$ \\
\hline Usuarios & $\begin{array}{l}\text { ¿La gerencia entiende que los principales usuarios de la información financiera son } \\
\text { los inversores, prestamistas y otros acreedores existentes o potenciales? }\end{array}$ & $\begin{array}{l}\text { Párrafo 1.5, } \\
\text { p. } 21\end{array}$ \\
\hline \multirow{3}{*}{$\begin{array}{l}\text { Cualidades } \\
\text { de la infor- } \\
\text { mación }\end{array}$} & $\begin{array}{l}\text { ¿La gerencia garantiza que la información financiera es relevante y presenta } \\
\text { fielmente lo que pretende representar? }\end{array}$ & $\begin{array}{l}\text { Párrafo } 2.4- \\
2.19, \text { pp. } 25-27\end{array}$ \\
\hline & $\begin{array}{l}\text { ¿La gerencia es responsable de que la información financiera influya en las deci- } \\
\text { siones tomadas por los usuarios y que la misma sea relevante? }\end{array}$ & \\
\hline & $\begin{array}{l}\text { ¿La gerencia vela por que la información financiera esté completa, sea neutral y } \\
\text { esté libre de error? }\end{array}$ & \\
\hline
\end{tabular}

Tabla 2. Categorías de la propuesta de marco conceptual para la información financiera 2015

\begin{tabular}{|c|c|c|}
\hline \multicolumn{3}{|c|}{ Propuesta de marco conceptual para la información financiera 2015 (IFRS, 2015) } \\
\hline Categoría & Pregunta(s) en la encuesta relacionada & $\begin{array}{l}\text { Página(s) } \\
\text { de } \\
\text { referencia }\end{array}$ \\
\hline \multirow{2}{*}{$\begin{array}{l}\text { Objetivo, } \\
\text { utilidad y } \\
\text { limitaciones de } \\
\text { la información } \\
\text { financiera }\end{array}$} & $\begin{array}{l}\text { ¿La gerencia debería ser responsable de evaluar las expectativas de los inversores, } \\
\text { prestamistas y otros acreedores sobre la rentabilidad y que la misma depende de } \\
\text { la administración que se haga de los recursos? }\end{array}$ & \multirow[t]{2}{*}{$\begin{array}{l}\text { Párrafo } 1.3 \\
1.4 \text { pp. } 21-22\end{array}$} \\
\hline & $\begin{array}{l}\text { ¿La gerencia debería proporcionar información sobre el uso que da a los recursos } \\
\text { de la entidad, ya que esto permite a los inversores, prestamistas y otros acreedores } \\
\text { evaluar cómo ha sido su gestión? }\end{array}$ & \\
\hline $\begin{array}{l}\text { Recursos } \\
\text { económicos y } \\
\text { derechos de } \\
\text { los acreedores }\end{array}$ & $\begin{array}{l}\text { ¿La gerencia debería proporcionar información sobre la naturaleza e importes de } \\
\text { los recursos económicos y los derechos de los acreedores, ya que esto permite a } \\
\text { los usuarios evaluar su gestión? }\end{array}$ & $\begin{array}{l}\text { Párrafo 1.13, } \\
\text { p. } 23\end{array}$ \\
\hline $\begin{array}{l}\text { Cambios en } \\
\text { los recursos }\end{array}$ & $\begin{array}{l}\text { ¿Los gerentes deberían revelar los cambios de los recursos económicos y en los } \\
\text { derechos de los acreedores para que los usuarios puedan evaluar su gestión? }\end{array}$ & $\begin{array}{l}\text { Párrafo } 1.15, \\
1.16, \text { p. } 23\end{array}$ \\
\hline $\begin{array}{l}\text { económicos } \\
\text { y en los dere- } \\
\text { chos de los } \\
\text { acreedores }\end{array}$ & $\begin{array}{l}\text { ¿La gerencia debería proporcionar información sobre la rentabilidad que ha pro- } \\
\text { ducido la entidad para que los usuarios puedan evaluar su gestión? }\end{array}$ & \\
\hline $\begin{array}{l}\text { Rendimiento } \\
\text { financiero } \\
\text { reflejado por } \\
\text { flujos de efecti- } \\
\text { vo pasados }\end{array}$ & $\begin{array}{l}\text { ¿La gerencia debería ser responsable de la información sobre los flujos de efectivo } \\
\text { de un periodo, lo que permite que los usuarios puedan evaluar su gestión? }\end{array}$ & $\begin{array}{l}\text { Párrafo 1.20, } \\
\text { p. } 24\end{array}$ \\
\hline $\begin{array}{l}\text { Información } \\
\text { sobre la } \\
\text { eficiencia y } \\
\text { eficacia del uso } \\
\text { de los recursos } \\
\text { de la entidad }\end{array}$ & $\begin{array}{l}\text { ¿La gerencia debería proteger los recursos de la entidad contra efectos desfavor- } \\
\text { ables de factores económicos, tales como cambios tecnológicos o en precios, y } \\
\text { asegurar que la entidad cumple con la legislación, la regulación y las disposiciones } \\
\text { contractuales que le sean aplicables? }\end{array}$ & $\begin{array}{l}\text { Párrafo } 1.22, \\
1.23, \text { p. } 24\end{array}$ \\
\hline
\end{tabular}


Tabla 3. Categorías de NIC 8. Sección 10. Políticas contables

\begin{tabular}{|c|c|c|}
\hline \multicolumn{3}{|c|}{ NIC 8. Sección 10. Políticas contables (IFRS Fundation, 2009) } \\
\hline Categoría & Pregunta(s) en la encuesta relacionada & $\begin{array}{l}\text { Página de } \\
\text { referencia }\end{array}$ \\
\hline $\begin{array}{l}\text { Responsabilidad } \\
\text { Elaboración de }\end{array}$ & $\begin{array}{l}\text { ¿La gerencia usa su juicio para desarrollar y aplicar políticas contables que sean } \\
\text { relevantes y fiables? }\end{array}$ & $\begin{array}{l}\text { Párrafo } 10.4 \\
\text { p. } 54\end{array}$ \\
\hline $\begin{array}{l}\text { Elaboración de } \\
\text { políticas con- } \\
\text { tables }\end{array}$ & $\begin{array}{l}\text { ¿La gerencia desarrolla políticas contables que sean relevantes y fiables en aus- } \\
\text { encia de una NIIF aplicable a la transacción, suceso o condición? }\end{array}$ & \\
\hline
\end{tabular}

Tabla 4. Categorías de las responsabilidades para la gerencia en NIIF para PYMES

\begin{tabular}{|c|c|c|}
\hline \multicolumn{3}{|c|}{ Responsabilidades para la gerencia en NIIF para PYMES (IFRS Fundation, 2009) } \\
\hline Categoría & Pregunta(s) en la encuesta relacionada & $\begin{array}{l}\text { Página(s) de } \\
\text { referencia }\end{array}$ \\
\hline $\begin{array}{l}\text { En el objetivo } \\
\text { de los estados } \\
\text { financieros }\end{array}$ & $\begin{array}{l}\text { ¿Los estados financieros preparados por la administración dan cuenta de la } \\
\text { responsabilidad en la gestión de los recursos que lleva a cabo la gerencia? }\end{array}$ & $\begin{array}{l}\text { Párrafo 2.3, } \\
\text { p. } 13\end{array}$ \\
\hline $\begin{array}{l}\text { En el cumplimiento } \\
\text { de las NIIF }\end{array}$ & $\begin{array}{l}\text { ¿La gerencia es quien establece si los estados financieros cumplen o no con } \\
\text { las NIIF y si esta norma puede entrar en conflicto con el objetivo de los estados } \\
\text { financieros de las PYMEs? }\end{array}$ & $\begin{array}{l}\text { Párrafos } 3.4 \text { y } \\
3.5, \text { p. } 24\end{array}$ \\
\hline \multirow[t]{2}{*}{$\begin{array}{l}\text { En hipótesis de } \\
\text { negocio en marcha }\end{array}$} & $\begin{array}{l}\text { ¿La gerencia evalúa la capacidad de la entidad para continuar funcionando en } \\
\text { el futuro mediante el análisis de los estados financieros? }\end{array}$ & $\begin{array}{l}\text { Párrafos } 3.8 \text { y } \\
3.9, \text { p. } 22-26\end{array}$ \\
\hline & $\begin{array}{l}\text { ¿La gerencia revela la existencia de incertidumbres significativas relativas } \\
\text { a sucesos o condiciones que puedan aportar dudas importantes sobre la } \\
\text { capacidad de la entidad de continuar como negocio financieramente viable? }\end{array}$ & \\
\hline $\begin{array}{l}\text { En otra información } \\
\text { a revelar }\end{array}$ & $\begin{array}{l}\text { ¿El gerente revela el efectivo en caja y bancos y los instrumentos de inversión } \\
\text { significativos mantenidos por la entidad que no están disponibles para ser } \\
\text { utilizados por esta? }\end{array}$ & $\begin{array}{l}\text { Párrafo } 7.21, \\
\text { p. } 44\end{array}$ \\
\hline $\begin{array}{l}\text { En información } \\
\text { sobre juicios }\end{array}$ & $\begin{array}{l}\text { ¿El gerente revela los juicios que realizó al aplicar las políticas contables que } \\
\text { tengan efecto más significativo sobre los importes reconocidos en los estados } \\
\text { financieros de la entidad? }\end{array}$ & $\begin{array}{l}\text { Párrafo 8.6, } \\
\text { p. } 46\end{array}$ \\
\hline $\begin{array}{l}\text { En selección y apli- } \\
\text { cación de políticas } \\
\text { contables }\end{array}$ & $\begin{array}{l}\text { ¿La gerencia reconoce y aplica los requerimientos, guías, definiciones, } \\
\text { criterios de reconocimiento y conceptos de medición para activos, pasivos, } \\
\text { ingresos y gastos establecidos en las NIIF y en el marco conceptual para la } \\
\text { información financiera? }\end{array}$ & $\begin{array}{l}\text { Párrafos 10.4, } \\
10.5,10.6, \\
\text { p. } 54\end{array}$ \\
\hline $\begin{array}{l}\text { En información a } \\
\text { revelar sobre las } \\
\text { remuneraciones } \\
\text { del personal clave } \\
\text { de la gerencia }\end{array}$ & $\begin{array}{l}\text { ¿La gerencia revela el total de las remuneraciones (beneficios de los } \\
\text { empleados) del personal clave de la gerencia (personas que tienen autoridad } \\
\text { y responsabilidad para planificar, dirigir y controlar las actividades de la enti- } \\
\text { dad, directa o indirectamente)? }\end{array}$ & $\begin{array}{l}\text { Párrafos } 33.6 \text { y } \\
33.7, \text { p. } 226\end{array}$ \\
\hline
\end{tabular}


Tabla 5. Categorías de los documentos NIIF comentarios de la gerencia

\begin{tabular}{|c|c|c|}
\hline \multicolumn{3}{|c|}{ Documento NIIF comentarios de la gerencia (IFRS Foundation, 2010) } \\
\hline Categoría & Pregunta(s) en la encuesta relacionada & $\begin{array}{l}\text { Página(s) de } \\
\text { referencia }\end{array}$ \\
\hline $\begin{array}{l}\text { Naturaleza } \\
\text { del negocio }\end{array}$ & $\begin{array}{l}\text { ¿La gerencia proporciona junto con los estados financieros un informe de gestión } \\
\text { escrito, con el propósito de ayudar a los usuarios de los informes financieros a } \\
\text { aumentar su comprensión de la entidad y del entorno externo en que opera? }\end{array}$ & $\begin{array}{l}\text { Párrafo 26, } \\
\text { p. } 10\end{array}$ \\
\hline \multirow[t]{2}{*}{$\begin{array}{l}\text { Objetivos y } \\
\text { estrategias }\end{array}$} & $\begin{array}{l}\text { ¿El informe de gestión que realiza la gerencia revela los objetivos y estrategias que } \\
\text { la misma usa en su administración de forma que permita a los usuarios mediante los } \\
\text { informes financieros comprender las prioridades de su acción, así como identificar los } \\
\text { recursos que deben gestionarse para proporcionar resultados? }\end{array}$ & $\begin{array}{l}\text { Párrafos 27- } \\
28, \text { pp. 10-11 }\end{array}$ \\
\hline & $\begin{array}{l}\text { ¿La gerencia analiza en el informe de gestión los cambios significativos en los } \\
\text { objetivos y estrategias de una entidad en relación con los periodos anteriores? }\end{array}$ & \\
\hline \multirow[t]{4}{*}{$\begin{array}{l}\text { Recursos, } \\
\text { riesgos y } \\
\text { relaciones }\end{array}$} & $\begin{array}{l}\text { ¿La gerencia revela en su informe de gestión una descripción clara de los recursos, } \\
\text { riesgos y relaciones más importantes que la gerencia considera que pueden afectar el } \\
\text { valor de la entidad y la forma en que se gestionan esos recursos, riesgos y relaciones? }\end{array}$ & $\begin{array}{l}\text { Párrafos 29- } \\
33, \text { p. } 11\end{array}$ \\
\hline & $\begin{array}{l}\text { ¿La gerencia revela en su informe de gestión los recursos financieros y no financieros } \\
\text { disponibles para la entidad que sean fundamentales para la misma? }\end{array}$ & \\
\hline & $\begin{array}{l}\text { ¿La gerencia revela en su informe de gestión las exposiciones a los riesgos principales } \\
\text { de una entidad y los cambios en esos riesgos, junto con sus planes y estrategias para } \\
\text { hacerles frente o mitigarlos, así como la eficacia de sus estrategias de gestión de riesgos? }\end{array}$ & \\
\hline & $\begin{array}{l}\text { ¿La gerencia revela en su informe de gestión las relaciones significativas que la entidad } \\
\text { tiene con grupos de interés, la forma en que esas relaciones están probablemente afectan- } \\
\text { do al rendimiento y valor de la entidad y la forma en que se gestionan esas relaciones? }\end{array}$ & \\
\hline \multirow[t]{3}{*}{$\begin{array}{l}\text { Resultados } \\
\text { y perspec- } \\
\text { tivas }\end{array}$} & $\begin{array}{l}\text { ¿La gerencia revela en su informe de gestión una descripción clara del rendimiento } \\
\text { financiero y no financiero de la entidad, la medida en que ese rendimiento puede ser } \\
\text { un indicador del rendimiento futuro y la evaluación de la gerencia de las perspectivas } \\
\text { de la entidad? }\end{array}$ & $\begin{array}{l}\text { Párrafos 34- } \\
36, \text { pp. 11-12 }\end{array}$ \\
\hline & $\begin{array}{l}\text { ¿La gerencia revela en su informe de gestión explicaciones del rendimiento y progreso } \\
\text { de la entidad durante el periodo y su situación al final de ese periodo? }\end{array}$ & \\
\hline & $\begin{array}{l}\text { ¿La gerencia explica los riesgos e hipótesis necesarias para que los usuarios evalúen la } \\
\text { probabilidad de conseguir los objetivos estratégicos cuando estos están cuantificados? }\end{array}$ & \\
\hline $\begin{array}{l}\text { Medidas de } \\
\text { rendimiento }\end{array}$ & $\begin{array}{l}\text { ¿La gerencia revela las medidas de rendimiento e indicadores (financieros y no } \\
\text { financieros en ambos casos) que utiliza para evaluar el progreso hacia los objetivos } \\
\text { establecidos? }\end{array}$ & $\begin{array}{l}\text { Párrafos } 37- \\
40, \text { p. } 12\end{array}$ \\
\hline
\end{tabular}

De esta forma, el cuestionario final estuvo compuesto en primera medida por 15 preguntas que se relacionan con la experiencia laboral y la preparación académica que se han tenido en relación con las NIIF; en segunda instancia, por 33 afirmaciones basadas en la categorización de los documentos medidas mediante la escala Likert de siete puntos: 1. Totalmente en desacuerdo, 2. En desacuerdo, 3. Moderadamente en desacuerdo, 4. Neutral, 5. Moderadamente de acuerdo, 6. De acuerdo y 7. Totalmente de acuerdo, y finalmente, 6 preguntas de carácter sociodemográfico. Como todo instrumento o método seleccionado para recolectar los datos se debe demostrar su confiabilidad y validez. Para 
lo anterior, se usó el método del coeficiente Alfa de Cronbach, sobre la base de la varianza de los ítems, los cuales indican una fiabilidad muy alta de la escala, por lo cual el instrumento es confiable para medir lo que se pretende en la investigación.

Sr. Autor, falta llamado de la tabla 6 en el texto

Tabla 6. Análisis de fiabilidad del instrumento

\begin{tabular}{ccc}
\hline Alfa de Cronbach & $\begin{array}{c}\text { Alfa de Cronbach basada en elementos } \\
\text { estandarizados }\end{array}$ & Número de elementos \\
\hline 0.951 & 0.952 & 33 \\
\hline
\end{tabular}

La validez de contenido de este instrumento se realizó consultando a tres doctores expertos académicos en contabilidad y auditoría, quienes fueron seleccionados por su gran experiencia y conocimiento de las NIIF para revisar y validar las preguntas.

\subsection{Aplicación del instrumento}

La aplicación del instrumento se realizó de manera física y por correo electrónico mediante el envío de un enlace de formulario de Google. Cada una de las preguntas suministradas en el cuestionario tuvo un carácter de obligatoriedad, lo que permitió que no quedara ninguna pregunta sin contestar antes de que se pudiera enviar el cuestionario. La aplicación del instrumento duró aproximadamente un año, mientras se obtenía un número adecuado de cuestionarios para lograr una muestra significativa. La muestra obtenida se compuso de 100 directivos de todos los niveles, que se encuentran actualmente trabajando en empresas de diferentes sectores, en toda Colombia.

\section{Resultados}

A continuación, se presentan las principales características de la muestra de los 100 directivos encuestados, para entender su composición y ver si podrían ayudar a explicar los resultados del estudio (tabla 7). 
Tabla 7. Características de la muestra

\begin{tabular}{|c|c|}
\hline Pregunta & Resultados \\
\hline ¿Cuál es su nivel de cargo? & $\begin{array}{l}\text { El } 57 \% \text { de ellos se encuentran en la dirección intermedia, el } 28 \% \text { en la alta dirección } \\
\text { y el } 15 \% \text { en la dirección de primera línea o dirección operativa. }\end{array}$ \\
\hline $\begin{array}{l}\text { ¿Sector económico a que } \\
\text { pertenece? }\end{array}$ & $\begin{array}{l}\text { La mayoría de los encuestados sostienen que pertenecen al sector de servicios } \\
\text { (44\%), el } 28 \% \text { pertenecen al sector de comercio, el } 9 \% \text { al sector de manufactura, el } \\
7 \% \text { a otro sector, el } 5 \% \text { al sector minero e hidrocarburos, el } 4 \% \text { al sector de construc- } \\
\text { ción, el } 2 \% \text { al sector agropecuario y el } 1 \% \text { al sector transporte. }\end{array}$ \\
\hline $\begin{array}{l}\text { ¿Antigüedad de la organiza- } \\
\text { ción? (Años de constituida) }\end{array}$ & $\begin{array}{l}\text { El } 50 \% \text { de los encuestados afirma que la antigüedad de la organización en donde } \\
\text { laboran es entre } 15 \text { y } 30 \text { años, el } 26 \% \text {, entre } 5 \text { y } 10 \text { años, el } 12 \% \text {, menos de } 5 \text { años y el } \\
12 \% \text { restante dicen que más de } 30 \text { años. }\end{array}$ \\
\hline $\begin{array}{l}\text { ¿Número de empleados que } \\
\text { tiene la organización? }\end{array}$ & $\begin{array}{l}\text { De acuerdo con los resultados, el } 49 \% \text { de los encuestados señalan que la organi- } \\
\text { zación tiene entre } 11 \text { y } 200 \text { empleados, el } 42 \% \text {, entre } 1 \text { y } 10 \text { empleados y el } 9 \% \text {, más } \\
\text { de } 200 \text { empleados. }\end{array}$ \\
\hline $\begin{array}{l}\text { Escriba en qué municipio se } \\
\text { encuentra ubicada la em- } \\
\text { presa en la que actualmente } \\
\text { ejerce funciones gerenciales. }\end{array}$ & $\begin{array}{l}\text { Teniendo en cuenta los resultados, el } 53 \% \text { de los encuestados afirman que la } \\
\text { empresa en que actualmente ejercen labores gerenciales se encuentra ubicada en } \\
\text { Bucaramanga, el } 13 \% \text { en Bogotá, el } 10 \% \text { en Floridablanca, el } 7 \% \text { en San Gil, el } 7 \% \\
\text { en Girón, el } 3 \% \text { en Piedecuesta, el } 2 \% \text { en varias ciudades, el } 2 \% \text { en Socorro, el } 1 \% \\
\text { en el Valle de San José, el } 1 \% \text { en Puerto Wilches y el } 1 \% \text { en Lebrija. }\end{array}$ \\
\hline $\begin{array}{l}\text { ¿La empresa que actual- } \\
\text { mente gerencia es de su } \\
\text { propiedad? }\end{array}$ & $\begin{array}{l}\text { El } 64 \% \text { de los encuestados manifiestan que la empresa donde actualmente geren- } \\
\text { cian no es de su propiedad, mientras que un } 36 \% \text { afirma que sí lo es. }\end{array}$ \\
\hline $\begin{array}{l}\text { ¿Ha recibido capacitación } \\
\text { en IFRS - NIIF (Normas } \\
\text { Internacionales de } \\
\text { Información Financiera)? }\end{array}$ & $\begin{array}{l}\text { El } 51 \% \text { de las personas encuestadas manifestaron que no han recibido capacitación } \\
\text { en IFRS - NIIF, mientras que el } 49 \% \text { aseguran que han recibido capacitación. }\end{array}$ \\
\hline $\begin{array}{l}\text { ¿Quién fue responsable de } \\
\text { la convergencia a NIIF en su } \\
\text { organización? }\end{array}$ & $\begin{array}{l}\text { Teniendo en cuenta los resultados, la opción que tuvo un mayor porcentaje de } \\
\text { respuestas sobre el responsable de la convergencia a NIIF en la organización fue la } \\
\text { del contador con un } 76 \% \text {. El } 49 \% \text { de las entidades utilizaron servicios de consultoría } \\
\text { externa, el } 30 \% \text { estuvo en cabeza de la revisoría fiscal, el } 11 \% \text { en cabeza del adminis- } \\
\text { trador, el } 6 \% \text { de la junta directiva, el } 5 \% \text { del auxiliar contable, el } 3 \% \text { de las gerencias } \\
\text { operativas y el } 1 \% \text { a cargo de la junta de socios. }\end{array}$ \\
\hline $\begin{array}{l}\text { ¿Quién estuvo a cargo } \\
\text { del diseño de las políticas } \\
\text { contables en la convergencia } \\
\text { a NIIF en su organización? }\end{array}$ & $\begin{array}{l}\text { El mayor porcentaje corresponde al contador con un } 77 \% \text {, seguido de las opciones } \\
\text { 'consultoría externa' con un } 45 \% \text {, 'revisoría fiscal' con un } 25 \% \text {, 'administrador' con } \\
\text { un } 14 \% \text {, 'auxiliar contable' con un } 5 \% \text {, 'junta directiva' con un } 4 \% \text {, 'junta de socios' } \\
\text { con un } 4 \% \text {, 'gerencias operativas' con un } 3 \% \text { y 'asamblea general de accionistas' con } \\
\text { un } 1 \% \text {. }\end{array}$ \\
\hline $\begin{array}{l}\text { ¿Qué áreas de la } \\
\text { organización se vieron } \\
\text { impactadas por la } \\
\text { convergencia a NIIF? }\end{array}$ & $\begin{array}{l}\text { Contabilidad con un } 87 \% \text { de respuestas, seguida por la de sistemas con un } 39 \% \text {, } \\
\text { control interno con un } 27 \% \text {, planeación financiera con un } 20 \% \text {, jurídica con un } 8 \% \text {, } \\
\text { recursos humanos con un } 6 \% \text { y comercial/ventas con un } 5 \% \text {. }\end{array}$ \\
\hline
\end{tabular}

A continuación, se presentan las medias de las 33 afirmaciones determinadas mediante la escala de Likert de 1 a 7 puntos, donde se observa que 22 de las mismas tienen una media por debajo de 4 puntos, lo cual indicaría que los gerentes tienden a estar en el rango 


\title{
de moderadamente en desacuerdo a neutral con afirmaciones que son su responsabilidad frente a las NIIF (tabla 8).
}

\author{
Tabla 8. Análisis descriptivo
}

\begin{tabular}{|c|c|}
\hline Afirmación & Media \\
\hline $\begin{array}{l}\text { 16.22. La gerencia revela en su informe de gestión una descripción clara del rendimiento financiero y } \\
\text { no financiero de la entidad, la medida en que ese rendimiento puede ser un indicador del rendimiento } \\
\text { futuro y la evaluación de la gerencia de las perspectivas de la entidad. }\end{array}$ & 2.49 \\
\hline $\begin{array}{l}\text { 16.24. La gerencia explica en su informe de gestión los riesgos e hipótesis necesarios para que los usuari- } \\
\text { os evalúen la probabilidad de conseguir los objetivos estratégicos cuando estos están cuantificados. }\end{array}$ & 2.58 \\
\hline $\begin{array}{l}\text { 16.8. La gerencia es quien establece si los estados financieros cumplen o no con las NIIF y si esta norma } \\
\text { puede entrar en conflicto con el objetivo de los estados financieros de las PYMES. }\end{array}$ & 2.62 \\
\hline $\begin{array}{l}\text { 16.14. La gerencia revela en notas a los estados financieros el total de las remuneraciones (beneficios de } \\
\text { los empleados) del personal clave de la gerencia (personas que tienen autoridad y responsabilidad para } \\
\text { planificar, dirigir y controlar las actividades de la entidad, directa o indirectamente). }\end{array}$ & 2.63 \\
\hline $\begin{array}{l}\text { 16.20. La gerencia revela en su informe de gestión las exposiciones a los riesgos principales de una enti- } \\
\text { dad y los cambios en esos riesgos, junto con sus planes y estrategias para hacerles frente o mitigarlos, así } \\
\text { como la eficacia de sus estrategias de gestión de riesgos. }\end{array}$ & 2.63 \\
\hline $\begin{array}{l}\text { 16.17. La gerencia analiza en el informe de gestión los cambios significativos en los objetivos y estrate- } \\
\text { gias de una entidad en relación con los periodos anteriores. }\end{array}$ & 2.70 \\
\hline $\begin{array}{l}\text { 16.18. La gerencia revela en su informe de gestión una descripción clara de los recursos, riesgos y rela- } \\
\text { ciones más importantes que la gerencia considera que pueden afectar al valor de la entidad y la forma } \\
\text { en que se gestionan esos recursos, riesgos y relaciones. }\end{array}$ & 2.80 \\
\hline $\begin{array}{l}\text { 16.16. El informe de gestión que realiza la gerencia revela los objetivos y estrategias que la misma usa en } \\
\text { su administración, de forma que permita a los usuarios mediante los informes financieros comprender } \\
\text { las prioridades de su acción, así como identificar los recursos que deben gestionarse para proporcionar } \\
\text { resultados. }\end{array}$ & 2.83 \\
\hline $\begin{array}{l}\text { 16.6. La gerencia desarrolla políticas contables relevantes y fiables, en ausencia de una NIIF aplicable a la } \\
\text { transacción, suceso o condición. }\end{array}$ & 2.88 \\
\hline $\begin{array}{l}\text { 16.25. La gerencia revela en su informe de gestión las medidas de rendimiento e indicadores (financieros } \\
\text { y no financieros en ambos casos) que utiliza para evaluar el progreso hacia los objetivos establecidos. }\end{array}$ & 2.91 \\
\hline $\begin{array}{l}\text { 16.10. La gerencia revela en notas a los estados financieros la existencia de incertidumbres significativas } \\
\text { relativas a sucesos o condiciones que puedan aportar dudas importantes sobre la capacidad de la enti- } \\
\text { dad de continuar como un negocio financieramente viable. }\end{array}$ & 2.92 \\
\hline $\begin{array}{l}\text { 16.5. La gerencia usa su juicio para desarrollar y aplicar políticas contables que sean relevantes y fiables y } \\
\text { estén acordes con las NIIF. }\end{array}$ & 3.02 \\
\hline $\begin{array}{l}\text { 16.7. Los estados financieros preparados por la administración dan cuenta de la responsabilidad en la } \\
\text { gestión de los recursos que lleva a cabo la gerencia. }\end{array}$ & 3.15 \\
\hline $\begin{array}{l}\text { 16.19. La gerencia revela en su informe de gestión los recursos financieros y no financieros disponibles } \\
\text { para la entidad que sean fundamentales para la misma. }\end{array}$ & 3.16 \\
\hline $\begin{array}{l}\text { 16.12. El gerente revela en notas a los estados financieros los juicios realizados al aplicar las políticas } \\
\text { contables que tengan efecto más significativo sobre los importes reconocidos en los estados financieros } \\
\text { de la entidad. }\end{array}$ & 3.34 \\
\hline $\begin{array}{l}\text { 16.21. La gerencia revela en su informe de gestión las relaciones significativas que la entidad tiene con } \\
\text { grupos de interés, la forma en que esas relaciones están probablemente afectando al rendimiento y valor } \\
\text { de la entidad y la forma en que se gestionan esas relaciones. }\end{array}$ & 3.34 \\
\hline
\end{tabular}




\section{Afirmación}

Media

16.23. La gerencia revela en su informe de gestión explicaciones del rendimiento y progreso de la enti-

dad durante el periodo y su situación al final de ese periodo.

17.8. La gerencia debería aplicar juicios sobre la materialidad en la preparación de los estados finan-

cieros y para ello debería considerar factores como los requerimientos de las NIIF, el conocimiento de los principales usuarios de la información financiera a presentar, la forma de organizar la información en los estados financieros y los estados financieros presentados anteriormente.

17.4. La gerencia debería proporcionar información sobre la naturaleza e importes de los recursos económicos y los derechos de los acreedores, ya que esto permite a los usuarios evaluar su gestión.

17.2. La gerencia debería ser la responsable de evaluar las expectativas de los inversores, prestamistas y

otros acreedores sobre la rentabilidad y asumir que la misma depende de la administración que se haga de los recursos de la entidad.

17.7. La gerencia debería ser responsable de la información sobre los flujos de efectivo de un periodo, lo $\quad 3.62$ cual permite que los usuarios puedan evaluar su gestión.

17.5. Los gerentes deberían revelar en notas a los estados financieros los cambios de los recursos económicos y en los derechos de los acreedores, para que los usuarios puedan evaluar su gestión.

17.6. La gerencia debería proporcionar información sobre la rentabilidad que ha producido la entidad, 4.02 para que los usuarios puedan evaluar su gestión.

17.3. La gerencia debería proporcionar información sobre el uso que da a los recursos de la entidad, ya $\quad 4.14$ que esto permite a los inversores, prestamistas y otros acreedores evaluar cómo ha sido su gestión.

17.1. La gerencia debería ser la responsable de proteger los recursos de la entidad contra efectos 4.26 desfavorables de factores económicos tales como cambios tecnológicos o en precios y asegurar que la entidad cumple con la legislación, regulación y las disposiciones contractuales que le sean aplicables.

16. 2. La gerencia, al preparar los estados financieros, tiene en cuenta principalmente las necesidades de 4.49 información de los inversores, prestamistas y otros acreedores existentes o potenciales.

16.9. La gerencia evalúa la capacidad de la entidad para continuar funcionando en el futuro mediante el $\quad 4.60$ análisis de los estados financieros.

16.13. La gerencia reconoce y aplica los requerimientos, guías, definiciones, criterios de reconocimiento y conceptos de medición para activos, pasivos, ingresos y gastos establecidos en las NIIF y en el marco conceptual para la información financiera.

16.1. La gerencia proporciona información financiera sobre la entidad que es útil a los inversores, prestamistas y otros acreedores existentes y potenciales para tomar decisiones sobre el suministro de recursos a la entidad.

16.4. La gerencia es responsable de que la información financiera sea fiable, es decir, que esté completa, sea neutral y esté libre de error.

16.15. La gerencia proporciona junto con los estados financieros un informe de gestión, con el propósito de ayudar a los usuarios de los informes financieros a aumentar su comprensión de la entidad y del entorno externo en que opera.

16.11. El gerente revela en notas a los estados financieros el efectivo en caja y bancos, y los instrumentos de inversión significativos mantenidos por la entidad que no están disponibles para ser utilizados por esta.

16.3. La gerencia garantiza que la información financiera es relevante, es decir, que la información financiera influye en las decisiones tomadas por los usuarios. 


\subsection{Análisis factorial}

En vista de la gran cantidad de variables objeto de estudio, se decidió aplicar un análisis factorial con el propósito de reducir los datos y encontrar grupos homogéneos de variables que ayudaran a explicar mejor las responsabilidades de la gerencia frente a las NIIF. Estos grupos homogéneos pero independientes entre sí se conocen como factores y resulta interesante saber si surgen variables nuevas con un significado común y ver a qué apunta este significado. Siguiendo este hilo, se aplicó un análisis factorial a las 33 variables objeto de estudio, con el método de extracción de componentes principales, utilizando una rotación varimax para una mejor visualización. De igual forma, se utilizó el test de esfericidad de Barlett para contrastar formalmente la existencia de correlación entre las variables, la cual resultó significativa (tablas 9 y 10).

Tabla 9.

\begin{tabular}{lll}
\hline \multicolumn{3}{c}{ Prueba de KMO y Bartlett } \\
\hline Medida Kaiser-Meyer-Olkin de adecuación de muestreo & 0.901 \\
\hline Prueba de esfericidad de Bartlett & Aprox. Chi-cuadrado & 3369.799 \\
\cline { 2 - 3 } & gl & 528 \\
\cline { 2 - 3 } & Sig. & 0.000 \\
\hline
\end{tabular}

Tabla 10. Análisis Factorial

\begin{tabular}{|c|c|c|c|c|c|}
\hline \multicolumn{6}{|c|}{ Componentes } \\
\hline Afirmaciones & 1 & 2 & 3 & 4 & 5 \\
\hline 16.5 & 0.776 & & & & \\
\hline 16.6 & 0.792 & & & & \\
\hline 16.7 & 0.735 & & & & \\
\hline 16.8 & 0.743 & & & & \\
\hline 16.10 & 0.861 & & & & \\
\hline 16.12 & 0.691 & & & & \\
\hline 16.14 & 0.871 & & & & \\
\hline 16.16 & 0.889 & & & & \\
\hline 16.17 & 0.895 & & & & \\
\hline 16.18 & 0.899 & & & & \\
\hline 16.19 & 0.841 & & & & \\
\hline 16.20 & 0.883 & & & & \\
\hline 16.21 & 0.770 & & & & \\
\hline
\end{tabular}




\begin{tabular}{|c|c|c|c|c|c|}
\hline \multicolumn{6}{|c|}{ Componentes } \\
\hline Afirmaciones & 1 & 2 & 3 & 4 & 5 \\
\hline 16.22 & 0.864 & & & & \\
\hline 16.23 & 0.692 & & & & \\
\hline 16.24 & 0.806 & & & & \\
\hline 16.25 & 0.776 & & & & \\
\hline 17.8 & 0.436 & & & & \\
\hline 16.3 & & 0.718 & & & \\
\hline 16.9 & & 0.810 & & & \\
\hline 16.11 & & 0.878 & & & \\
\hline 16.13 & & 0.844 & & & \\
\hline 16.15 & & 0.853 & & & \\
\hline 17.2 & & & 0.677 & & \\
\hline 17.4 & & & 0.720 & & \\
\hline 17.5 & & & 0.746 & & \\
\hline 17.1 & & & & 0.702 & \\
\hline 17.3 & & & & 0.732 & \\
\hline 17.6 & & & & 0.786 & \\
\hline 17.7 & & & & 0.560 & \\
\hline 16.1 & & & & & 0.599 \\
\hline 16.2 & & & & & 0.736 \\
\hline 16.4 & & & & & 0.601 \\
\hline
\end{tabular}

El análisis factorial arrojó la conformación de 5 factores, que en su conjunto explican el 75.8\% de la varianza, los cuales se muestran en la Tabla 10, donde los números al lado izquierdo de cada variable muestran el número de la pregunta en el cuestionario final. Cada uno de los 5 factores tuvo un Alfa de Cronbach de más de $80 \%$. Es interesante analizar las variables que agrupa cada factor, pues deja ver una clasificación de las responsabilidades de la gerencia frente a las NIIF, las cuales han sido llamadas a criterio de los autores de la siguiente manera:

Factor 1. Responsabilidad en el cumplimiento de objetivos, administración de riesgos y definición de políticas contables

Factor 2. Responsabilidad en la correcta aplicación de las NIIF y el marco conceptual

Factor 3. Responsabilidad en el cumplimiento de expectativas financieras de los usuarios 


\title{
Factor 4. Responsabilidad frente a la gestión realizada de los recursos suministrados
}

\author{
Factor 5. Responsabilidad en la presentación de información financiera útil
}

\author{
A continuación, en las tablas 11, 12, 13, 14 y 15 se presenta cada factor con sus correspon- \\ dientes variables y se otorga una explicación aproximada del porqué de la agrupación dada.
}

Tabla 11.

Factor 1. Responsabilidad en el cumplimiento de objetivos, administración de riesgos Media y definición de políticas contables

16.22. La gerencia revela en su informe de gestión una descripción clara del rendimiento financiero y 2.49 no financiero de la entidad, la medida en que ese rendimiento puede ser un indicador del rendimiento futuro y la evaluación de la gerencia de las perspectivas de la entidad.

16.24. La gerencia explica en su informe de gestión los riesgos e hipótesis necesarias para que los usuari- 2.58 os evalúen la probabilidad de conseguir los objetivos estratégicos cuando estos están cuantificados.

16.8. La gerencia es quien establece si los estados financieros cumplen o no con las NIIF y si esta norma 2.62 puede entrar en conflicto con el objetivo de los estados financieros de las PYMEs.

16.14. La gerencia revela en notas a los estados financieros el total de las remuneraciones (beneficios de los empleados) del personal clave de la gerencia (personas que tienen autoridad y responsabilidad para planificar, dirigir y controlar las actividades de la entidad, directa o indirectamente).

16.20. La gerencia revela en su informe de gestión las exposiciones a los riesgos principales de una enti-

dad y los cambios en esos riesgos, junto con sus planes y estrategias para hacerles frente o mitigarlos, así como la eficacia de sus estrategias de gestión de riesgos.

16.17. La gerencia analiza en el informe de gestión los cambios significativos en los objetivos y estrate- 2.70 gias de una entidad en relación con los periodos anteriores.

16.18. La gerencia revela en su informe de gestión una descripción clara de los recursos, riesgos y rela2.80 ciones más importantes que la gerencia considera que pueden afectar el valor de la entidad y la forma en que se gestionan esos recursos, riesgos y relaciones.

16.16. El informe de gestión que realiza la gerencia revela los objetivos y estrategias que la misma usa en su administración, de forma que permita a los usuarios mediante los informes financieros comprender las prioridades de su acción, así como identificar los recursos que deben gestionarse para proporcionar resultados.

16.6. La gerencia desarrolla políticas contables relevantes y fiables, en ausencia de una NIIF aplicable a la 2.88 transacción, suceso o condición.

16.25. La gerencia revela en su informe de gestión las medidas de rendimiento e indicadores (financieros 2.91 y no financieros en ambos casos) que utiliza para evaluar el progreso hacia los objetivos establecidos.

16.10. La gerencia revela en notas a los estados financieros la existencia de incertidumbres significativas 2.92 relativas a sucesos o condiciones, que puedan aportar dudas importantes sobre la capacidad de la entidad de continuar como un negocio financieramente viable.

16.5. La gerencia usa su juicio para desarrollar y aplicar políticas contables que sean relevantes y fiables y 3.02 estén acordes con las NIIF.

16.7. Los estados financieros preparados por la administración dan cuenta de la responsabilidad en la 3.15 gestión de los recursos que lleva a cabo la gerencia. 
Factor 1. Responsabilidad en el cumplimiento de objetivos, administración de riesgos y definición de políticas contables

16.19. La gerencia revela en su informe de gestión los recursos financieros y no financieros disponibles para la entidad que sean fundamentales para la misma.

16.12. El gerente revela en notas a los estados financieros los juicios realizados al aplicar las políticas

contables que tengan efecto más significativo sobre los importes reconocidos en los estados financieros de la entidad.

16.21. La gerencia revela en su informe de gestión las relaciones significativas que la entidad tiene con grupos de interés, la forma en que esas relaciones están probablemente afectando el rendimiento y valor de la entidad y la forma en que se gestionan esas relaciones.

16.23. La gerencia revela en su informe de gestión explicaciones del rendimiento y progreso de la entidad durante el periodo y su situación al final de ese periodo.

17.8. La gerencia debería aplicar juicios sobre la materialidad en la preparación de los estados financieros 3.44 y para ello debería considerar factores como los requerimientos de las NIIF, el conocimiento de los principales usuarios de la información financiera a presentar, la forma de organizar la información en los estados financieros y los estados financieros presentados anteriormente.

Con un Alfa de Cronbach de 0.973 , el factor 1 agrupa 18 variables, cuyo promedio ponderado es de 2.93. De acuerdo con la escala de Likert, la opción 3 corresponde a moderadamente en desacuerdo, lo cual significaría que los gerentes encuestados no están de acuerdo con revelar información relacionada con el cumplimiento de objetivos reflejados en el rendimiento financiero y no financiero de la entidad; también están moderadamente en desacuerdo con revelar las estrategias utilizadas para mitigar riesgos y definir políticas contables, además de no asumir su responsabilidad en la preparación de estados financieros acordes con las NIIF.

Hay que recordar en este punto que en la pregunta sobre quién definió las políticas contables en la convergencia, el $76 \%$ de los casos fue el contador, lo cual contraría notablemente la NIC 8 subsección 10 de políticas contables, donde esta responsabilidad cae en cabeza del administrador.

\section{Tabla 12.}

Factor 2. Responsabilidad en la correcta aplicación de las NIIF y el marco conceptual Media

16.9. La gerencia evalúa la capacidad de la entidad para continuar funcionando en el futuro mediante el $\quad 4.60$ análisis de los estados financieros.

16.13. La gerencia reconoce y aplica los requerimientos, guías, definiciones, criterios de reconocimiento 4.63 y conceptos de medición para activos, pasivos, ingresos y gastos establecidos en las NIIF y en el marco conceptual para la información financiera. 
Factor 2. Responsabilidad en la correcta aplicación de las NIIF y el marco conceptual Media

16.15. La gerencia proporciona junto con los estados financieros un informe de gestión, con el propósito

de ayudar a los usuarios de los informes financieros a aumentar su comprensión de la entidad y del entorno externo en que opera.

16.11. El gerente revela en notas a los estados financieros el efectivo en caja y bancos, y los instrumentos 5.14 de inversión significativos mantenidos por la entidad que no están disponibles para ser utilizados por esta.

16.3. La gerencia garantiza que la información financiera es relevante, es decir, que la información finan5.55 ciera influye en las decisiones tomadas por los usuarios.

Con un Alfa de Cronbach de 0.911, el factor 2 está conformado por 5 variables, cuyo promedio ponderado es de 5.01. De acuerdo con la escala de Likert, 5 corresponde a moderadamente de acuerdo, lo cual significa que los gerentes entienden su responsabilidad de aplicar las NIIF y el marco conceptual y su relación con la hipótesis de negocio en marcha, además de garantizar que la información suministrada sea relevante y fiable, lo cual puede mejorarse aún más si los estados financieros se acompañan de un informe de gestión. No obstante, como se pudo observar en el factor 1, al parecer la información que los gerentes consideran debería ir en dicho informe no coincide con el documento NIIF comentarios de la gerencia o no conocen el documento, el cual propone una estructura de revelación clara, completa y transparente.

\section{Tabla 13.}

\section{Factor 3. Responsabilidad en el cumplimiento de expectativas financieras de los usuarios Media}

17.4. La gerencia debería proporcionar información sobre la naturaleza e importes de los recursos 3.46 económicos y los derechos de los acreedores, ya que esto permite a los usuarios evaluar su gestión.

17.2. La gerencia debería ser la responsable de evaluar las expectativas de los inversores, prestamistas y

Con un Alfa de Cronbach 0.804 , el factor 3 reúne 3 variables, todas relacionadas con la propuesta del marco conceptual para la información financiera de utilizar las NIIF como instrumento de evaluación de la gerencia, al poder evaluar la administración realizada de los recursos de la entidad, en el sentido de proporcionar información sobre los recursos económicos y los derechos de los acreedores, y si el manejo de los mismos satisface las expectativas de los usuarios principales. En este punto, con un promedio de 3.57 los gerentes están en un rango 
de moderadamente en desacuerdo (3) a neutral (4), lo cual es preocupante, ya que la gerencia debe hacerse responsable de su gestión y rendir cuentas para evitar los conflictos de agencia.

Tabla 14.

Factor 4. Responsabilidad frente a la gestión realizada de los recursos suministrados Media

17.7. La gerencia debería ser responsable de la información sobre los flujos de efectivo de un periodo, lo 3.62 cual permite que los usuarios puedan evaluar su gestión.

17.6. La gerencia debería proporcionar información sobre la rentabilidad que ha producido la entidad, $\quad 4.02$ para que los usuarios puedan evaluar su gestión.

17.3. La gerencia debería proporcionar información sobre el uso que da a los recursos de la entidad, ya 4.14 que esto permite a los inversores, prestamistas y otros acreedores evaluar cómo ha sido su gestión.

17.1. La gerencia debería ser la responsable de proteger los recursos de la entidad contra efectos 4.26 desfavorables de factores económicos tales como cambios tecnológicos o en precios y asegurar que la entidad cumple con la legislación, regulación y disposiciones contractuales que le sean aplicables.

Con un Alfa de Cronbach de 0.829, el factor 4 se conformó por 4 variables, que agrupan los aspectos relacionados con la propuesta del marco conceptual de utilizar la información financiera para evaluar cómo fue el uso de los recursos de la entidad por parte de la gerencia, en términos de rentabilidad y flujo de caja, y si su administración busca proteger los recursos de situaciones que puedan generar riesgos e impedir el cumplimiento del objetivo financiero de maximizar el valor. A pesar de la similitud de este factor al 3, es importante mencionar que una cosa es evaluar la gestión realizada por la administración y otra muy diferente que la misma satisfaga las expectativas de los inversores, ya que la gestión pudo ser conservadora, pero los deseos de los inversores iban en otra dirección. Por ello se entiende la separación de los factores 3 y 4.

Tabla 15.

Factor 5. Responsabilidad en la presentación de información financiera útil Media

16. 2. La gerencia, al preparar los estados financieros, tiene en cuenta principalmente las necesidades de 4.49 información de los inversores, prestamistas y otros acreedores existentes o potenciales. 16.1. La gerencia proporciona información financiera sobre la entidad que es útil a los inversores, presta-
mistas y otros acreedores existentes y potenciales para tomar decisiones sobre el suministro de recursos a 4.88 la entidad.

16.4. La gerencia es responsable de que la información financiera sea fiable, es decir, que esté completa, 4.88 sea neutral y esté libre de error.

Con un Alfa de Cronbach de 0.836 el factor 5 agrupó 3 variables, todas relacionadas con las características cualitativas de la información financiera: que sea relevante y fiable 
para que sea realmente útil en la toma de decisiones. Recordemos que la relevancia es la capacidad que tiene la información de influir en la toma de decisiones por su presencia o ausencia y aquí la gerencia es quien debe ejercer el juicio teniendo en cuenta las necesidades de los usuarios. Este factor tuvo un promedio de 4.75, que para efectos de la escala de Likert se acerca a moderadamente de acuerdo según la percepción de los gerentes encuestados.

\section{Discusión de resultados}

Los resultados obtenidos a partir de la realización del cuestionario evidencian que los gerentes están moderadamente en desacuerdo con su responsabilidad frente a revelación del cumplimiento de objetivos, la administración de riesgos y la definición de políticas contables. Lo anterior podría sugerir un desvío de la misión de los gerentes en relación con el objetivo de las NIIF de suministrar información útil a los usuarios, además de evidenciar posibles debilidades de gobierno corporativo, control interno y delegación indebida de funciones en las empresas.

Lo anterior, quedó evidenciado al delegar a los contadores la responsabilidad de la convergencia a NIIF y la definición de las políticas contables, lo cual fue un error, pues es el gerente quien conoce los objetivos estratégicos del negocio y sus principales riesgos, aspectos fundamentales a tener en cuenta cuando se definen políticas contables en NIIF. Como lo afirma Ferrer (2013), cuando la contabilidad no se concibe como un sistema y la información financiera en las empresas no incluye aspectos que se relacionan con el análisis gerencial, estratégico y político de la compañía no existe un entendimiento del negocio por parte de los contadores y otras áreas de la empresa, y a su vez se presentan deficiencias en la conexión que debe existir en la empresa.

En cuanto a la responsabilidad en la correcta aplicación de las NIIF y el marco conceptual que se derive de la presentación de información financiera útil, los encuestados están de acuerdo con presentar información relevante y fiable al preparar estados financieros y proporcionar junto con los mismos un informe de gestión, pero no existe una 
vinculación entre los estados financieros preparados por la administración y el informe de gestión presentado; además, los gerentes no están de acuerdo con ser responsables de que los estados financieros cumplan con las NIIF. Adicionalmente, no consideran que sea parte de su responsabilidad el revelar incertidumbres significativas ni los juicios realizados para aplicar las políticas contables ni el total de remuneraciones del personal clave de la gerencia.

En cuanto a la responsabilidad en el cumplimiento de expectativas financieras de los usuarios y su gestión realizada de los recursos económicos, los gerentes no están de acuerdo con su responsabilidad de revelar los objetivos y las estrategias de su administración; de analizar los cambios significativos sobre los mismos; de dar una descripción clara de los recursos, riesgos y relaciones más importantes ni de dar explicaciones del rendimiento y el progreso de la entidad, mediante medidas de rendimiento e indicadores que se utilicen para evaluar el progreso de los objetivos. A propósito de ello, Higuera (2016) afirma que los estados financieros bajo las NIIF no pueden limitarse a ofrecer datos de carácter cuantitativo, estos deben además contener información sobre las políticas, objetivos, estrategias, procedimientos y manejo del capital de carácter cualitativo, de tal forma que los usuarios puedan conocer los riesgos financieros, así como los conflictos de intereses, el riesgo moral y la gestión de la gerencia.

Adicionalmente, manifestaron desacuerdo en que la gerencia deba ser responsable de evaluar las expectativas de los inversores prestamistas y otros acreedores sobre la rentabilidad, mediante la revelación en notas de los cambios de los recursos económicos para que los usuarios puedan evaluar su gestión. Esto refleja en parte, los resultados obtenidos en la encuesta mundial de gerencia realizada por el Banco Mundial, en donde se encontró que los gerentes colombianos tienen una autopercepción muy alta frente a sus capacidades, pero ocupan el último lugar en Suramérica en relación con su calidad como gerentes (Departamento Nacional de Planeación, 2015). Esto indica que no existe un reconocimiento de los gerentes en Colombia sobre la necesidad de conocer con claridad acerca de las NIIF ni revelar cierto tipo de información importante a los usuarios, para que estos puedan evaluar la administración de los recursos llevada a cabo por los gerentes en las empresas. 


\section{Conclusiones}

El objetivo de la investigación partió de establecer la percepción de 100 gerentes de empresas colombianas respecto al grado de aplicación de las NIIF como instrumento de gestión empresarial, además de evaluar si están de acuerdo en que estas se utilicen para controlar la gestión de los recursos y el cumplimiento de expectativas de los principales usuarios de la información financiera. Esto fue reflejado mediante el planteamiento de dos hipótesis fundamentales. En este sentido, se descarta la hipótesis alternativa (1) y se mantiene la hipótesis nula (1) que manifiesta que los directivos, accionistas y personal encargado del área gerencial, contable y financiero de empresas no hacen aplicables las NIIF, en sus procesos de gestión empresarial. Asimismo, se descarta la hipótesis alternativa (2) y se mantiene la hipótesis nula (2) que sostiene que los gerentes no están de acuerdo con el objetivo propuesto por el IASB de que las NIIF sirvan para evaluar la gestión de los recursos hecha por la administración.

Finalmente, cabe preguntarse por las razones de esta situación, ¿podría ser desconocimiento de las responsabilidades frente a las NIIF por parte de los gerentes? ¿O tal vez se trata una delegación indebida de funciones al contador? ¿Será que faltan estrategias para generar y difundir las NIIF con un enfoque más gerencial que contable por parte de los reguladores, universidades y auditores? O ¿Será esto un reflejo de la ausencia de una cultura empresarial en Colombia que tienda a la transparencia, crecimiento sostenible y generación de valor, mediante la revelación de información financiera relevante y fiable? No nos atrevemos a dar razones a los hallazgos encontrados, pero es importante que el problema se evidencie y que el estudio se replique a una muestra mayor de directivos, de tal forma que se pueda buscar una solución, pues la sostenibilidad empresarial colombiana podría enfrentar riesgos importantes de asimetría de información financiera en un futuro cercano si esta es la situación de todos o de la mayoría de los gerentes colombianos. 


\section{Referencias}

Barrios, M. (2015). Superposición de roles gerenciales y roles propietarios en las pequeñas y medianas empresas familiares. Palermo Business Review, 7-60.

Consejo de Normas Internacionales de Contabilidad. (2015). Proyecto de norma. Marco conceptual para la información financiera. Londres: Autor.

Departamento Nacional de Planeación. (2015). Políticas de desarrollo productivo, ciencia, tecnología e innovación. Recuperado de https://minciencias.gov.co/sites/default/files/ ckeditor_files/borradorconpes-politicactei-2015-2025.pdf

Ferrer, A. (2013). Análisis del proceso de convergencia a Normas Internacionales de Contabilidad e Información Financiera desde los factores intrínsecos al sistema contable en Colombia. Cuadernos de Contabilidad, 14(36), 971-1007. Recuperado de http://revistas.javeriana.edu.co/index.php/cuacont/article/viewFile/7910/6293

Hernández, R., Fernández, C., \& Baptista, P. (2010). Metodología de la Investigación. México: Mc Graw Hill.

IFRS Foundation (2009). Módulo 2. Conceptos y Principios Generales. Londres: Consejo de Normas Internacionales de Contabilidad.

IFRS Foundation (2010). Conceptual Framework for Financial Reporting 2010. Londres: Consejo de Normas Internacionales de Contabilidad.

IFRS Foundation (2015). Proyecto de Norma Marco Conceptual para la Información Financiera. Londres: Consejo de Normas Internacionales de Contabilidad. Recuperado de https://www.ifrs.org/-/media/project/conceptual-framework/exposure-draft/published-documents/ed-conceptual-framework-es.pdf

Higuera, J. (2016). Convergencia a las NIIF: un cambio hacia la competitividad. Boletín de Coyuntura. Recuperado de http://www.fce.unal.edu.co/media/files/boletines/uamf/boletin6/index.html

Hunt, S. (1996). Modern marketing theory: Critical issues in the philosophy of marketing science. Nashville: South-Western Publications.

Jiménez, A. (2010). Los procesos de globalización e integración económica: retos y oportunidades para el mercadeo internacional de las empresas. Revista Venezolana de Análisis de Coyuntura, 16(2), 95-113. Recuperado de https://www.redalyc.org/comocitar. oa?id=36418856005

Lozano, M. B., Hidalgo, A. d., \& Pindado, J. (2004). El conflicto accionista-directivo: problemas y propuestas de solución. Tribuna de economía, 225-245. Recuperado de http://www. revistasice.com/index.php/ICE/article/view/624? 
Ocampo, P., \& Astudillo, R. (2015). Retos y alternativas gerenciales en la implementación de las NIC-NIIF en las pymes colombianas. Magazín Empresarial, 11(26), 11-19. Recuperado de http://revistas.usc.edu.co/index.php/magazin/article/view/594/484\#.WbgMA8gjHIU

Pineda, D. (2013). Importancia de la intervención de la administración en el proceso de transición a NIIF en las Pymes de Sogamoso, Boyacá. Revista de investigación, desarrollo e innovación, 3(2), 58-71. https://doi.org/10.19053/20278306.2167

Quintero, J. y Pinzón, N. (2016). Normas internacionales de información financiera (NII) en Colombia: mitigando el impacto del Earnings Management en los estados financieros. (Tesis de maestría, Colegio de Estudios Superiores de Administración - CESA). Recuperado de http://repository.cesa.edu.co/handle/10726/1564

Santos, G. (2015). Responsabilidad de los administradores en la implementación de las IFRS en Colombia. Revista Supersociedades, (10), 38-39. Recuperado de http://www.supersociedades.gov.co/prensa/Documentos_publicaciones/revista\%20supersociedades $\% 20$ V10\%20AV18\%202015.pdf 